\title{
Peritoneal Carcinomatosis from Appendiceal Cancer: Early Adequate Therapeutic Management for Long-Term Survival
}

\author{
Olivier Glehen, MD, PhD, Naoual Bakrin, MD, and Guillaume Passot, MD \\ Surgical Oncology Department, Centre Hospitalo-Universitaire Lyon Sud, Lyon, France
}

Epithelial appendiceal cancers are unusual, and nearly all patients with these tumors have peritoneal dissemination at the time of diagnosis. ${ }^{1}$ Most of them have no lymphatic or hematogeneous metastases, as has been reported by Chua et al. ${ }^{2}$ The site of treatment failure included the abdomen alone in $48 \%$ of patients, and only $4 \%$ developed distant metastatic site alone. Thus, there is a strong rationale for extensive locoregional treatment combining cytoreductive surgery and perioperative intraperitoneal chemotherapy to treat a locoregional disease. Long-term survival has already been reported for peritoneal dissemination of various etiologies treated with this aggressive but comprehensive combined therapeutic approach. ${ }^{3}$ Among all these etiologies, peritoneal carcinomatosis from appendiceal cancers represents one with the best prognosis, with 5-year survival rates ranging 45-65\% with appropriate therapeutic management. ${ }^{2,3}$ Elias et al., in a French multi-institutional study, demonstrated that locoregional treatment for appendiceal cancers was better than for colon, rectum, and small bowel adenocarcinoma. ${ }^{4}$ The characteristics and the extent of the disease were similar to those of tumors of other origins, signifying that the diagnosis of carcinomatosis from appendiceal tumors was not earlier. The site of recurrence was not recorded in their study. The prognosis difference in favor of appendiceal cancers may be explained by a lower risk of systemic or metastatic dissemination, as well as a possible different biologic behavior. Regarding the interesting longterm survival results already reported and confirmed by Chua et al. for this unusual disease, the combined approach

(C) Society of Surgical Oncology 2011

Published Online: 13 April 2011

O. Glehen, $\mathrm{MD}, \mathrm{PhD}$

e-mail: olivier.glehen@chu-lyon.fr should become the gold standard treatment of peritoneal carcinomatosis from appendiceal cancer. ${ }^{2}$

Another important issue from the recent report of Chua et al. is the negative prognostic impact of previous debulking surgery and/or neoadjuvant systemic chemotherapy. ${ }^{2}$ In patients that had not undergone oncological surgical treatment, the median survival was not reached, and the 5 -year survival rate was $75 \%$, whereas they were only 19.8 months and $20 \%$, respectively, for patients undergoing limited resection, and 33.5 months and $35 \%$, respectively, for patients undergoing debulking surgery. The peritoneum constitutes the first line of defense against carcinomatosis. Whenever the peritoneum is violated by surgery, residual cancer cells are implanted and then progress beyond the peritoneum. With this so-called cancercell entrapment, the adequate complete cytoreductive surgery and its peritonectomy procedures become technically much more difficult and less likely to be complete, leading to worse oncologic and long-term results. Moreover, tumor growth deep in the peritoneum at crucial anatomic sites such as ureters or big vessels increases the likelihood of injuries and severe postoperative complications. The relative sparing of the small bowel is only seen early on in the natural history of peritoneal carcinomatosis. After several or extensive surgical procedures have been performed, the fibrous adhesions that inevitably result are infiltrated by tumor. This lead to full-thickness involvement of the small bowel by the malignant process. Eventually, it becomes impossible to cytoreduce the tumor safely, and the effects of intraperitoneal chemotherapy by itself are not adequate to keep the patient free of disease. Previous extensive surgery also induces the formation of scars and/or fibrosis tissues. During cytoreduction procedure, it is difficult for the surgeon to see the difference between scar tissue and malignant tissue. For an adequate complete cytoreductive surgery that constitutes the principal prognostic factor, the surgeon may be obliged to perform a more extensive 
procedure, with higher postoperative risks, to remove all suspect tissues.

The negative impact of debulking surgery or incomplete cytoreductive surgery has already been demonstrated or reported for other peritoneal surface malignancies. For pseudomyxoma peritonei, the prior surgical score that quantify the extent of previous surgery has a strong prognostic influence for long-term survival. ${ }^{5}$ It was also suggested for colorectal carcinomatosis with better survival results in group of patients that were surgically treated for their primary tumor and carcinomatosis during the same procedure. ${ }^{6}$ More recently, for ovarian carcinomatosis, the definition of optimal cytoreductive surgery has evolved, and most authors consider that "optimal" should signify macroscopically complete removal of disease, not residual tumor nodules of less than $1-2 \mathrm{~cm}^{7,8}$

In conclusion, for carcinomatosis from appendiceal cancer and various origins, the adequate and early therapeutic management combining complete cytoreductive surgery and intraperitoneal chemotherapy will achieve better oncologic outcomes. The negative impact of inadequate previous debulking surgery should be underlined and disseminated to general surgeons and oncologists. Peritoneum should be respected as much as possible in order to not jeopardize future and adequate procedures that may be delivered with curative intent.

\section{REFERENCES}

1. Sugarbaker PH. New standard of care for appendiceal epithelial neoplasms and pseudomyxoma peritonei syndrome? Lancet Oncol. 2006;7:69-76.

2. Chua TC, Al-Alem I, Saxena A, Liauw W, Morris D. Surgical cytoreduction and survival in appendiceal cancer peritoneal carcinomatosis: an evaluation of 46 consecutive patients. Ann Surg Oncol. In press.

3. Glehen O, Gilly FN, Boutitie F, et al. Toward curative treatment of peritoneal carcinomatosis from nonovarian origin by cytoreductive surgery combined with perioperative intraperitoneal chemotherapy: a multi-institutional study of 1,290 patients. Cancer. 2010;116:5608-18.

4. Elias D, Glehen O, Pocard M, et al. A comparative study of complete cytoreductive surgery plus intraperitoneal chemotherapy to treat peritoneal dissemination from colon, rectum, small bowel, and nonpseudomyxoma appendix. Ann Surg. 2010;251:896-901.

5. Sugarbaker PH, Chang D. Results of treatment of 385 patients with peritoneal surface spread of appendiceal malignancy. Ann Surg Oncol. 1999;6:727-31.

6. Pestieau SR, Sugarbaker PH. Treatment of primary colon cancer with peritoneal carcinomatosis: comparison of concomitant vs. delayed management. Dis Colon Rectum. 2000;43:1341-6.

7. Bristow RE, Tomacruz RS, Armstrong DK, Trimble EL, Montz FJ. Survival effect of maximal cytoreductive surgery for advanced ovarian carcinoma during the platinum era: a meta-analysis. J Clin Oncol. 2002;20:1248-59.

8. Gultekin M, Dursun P, Dogan NU, et al. Debulking surgery for incompletely operated advanced epithelial ovarian carcinoma. $J$ Surg Oncol. 2009;100:258-60. 